\title{
Seizure and Coma Following Kratom (Mitragynina speciosa Korth) Exposure
}

\author{
Jamie L. Nelsen • Jeff Lapoint • Michael J. Hodgman • \\ Kenneth M. Aldous
}

Published online: 22 April 2010

(C) American College of Medical Toxicology 2010

\begin{abstract}
Reports of toxicity secondary to Kratom are rare and lack of diagnostic testing in human specimens has prevented confirmatory explanation of observed clinical effects. We present a novel case of serious human toxicity following Kratom use confirmed via quantitative analysis of urine by high performance liquid chromatography coupled to electrospray tandem mass spectrometry. A 64 year-old male was witnessed to have a seizure at home following kratom consumption. Upon arrival to the emergency department (ED), the patient was unresponsive. While in the ED, the patient sustained a second seizure. He was intubated to protect
\end{abstract}

This report was presented as a Platform Presentation: NACCT 2009, San Antonio, TX. AACT's SIG on Herbs and Dietary Supplements.

J. L. Nelsen $(\square)$

Department of Emergency Medicine,

SUNY Upstate Medical University,

Upstate New York Poison Center,

250 Harrison Street,

Syracuse, NY 13210, USA

e-mail: nelsenj@upstate.edu

\section{J. Lapoint}

Department of Emergency Medicine,

SUNY Upstate Medical University,

750 E. Adams Street,

Syracuse, NY, USA

M. J. Hodgman

Department of Emergency Medicine,

Basset Health Care,

One Atwell Road,

Cooperstown, NY, USA

\section{K. M. Aldous}

Division of Environmental Health Sciences,

New York State Department of Health,

Wadsworth Center, Empire State Plaza,

P.O. Box 509, Albany, NY 12201-0509, USA his airway. The remainder of his hospital course was uneventful. A urine specimen was collected shortly after admission and sent for analysis. The mitragynine concentration in the urine was $167 \pm 15 \mathrm{ng} / \mathrm{ml}$. We report a rare case of Kratom toxicity characterized by a seizure and coma confirmed by urinary analysis of mitragynine by high performance liquid chromatography coupled to electrospray tandem mass spectrometry. The proposed mechanism for this reaction is unclear but suggested mechanisms include adenosine binding or stimulation of adrenergic and/or serotonergic receptors similar to tramadol.

Keywords Kratom · Mitragynina speciosa Korth · Seizure · Coma

\section{Introduction}

Mitragyna speciosa Korth, commonly known as kratom, is an herbal drug that has activity at opioid receptors and has been promoted as an adjunct to chronic pain therapy, a drug of abuse, as well as a means to reduce opioid tolerance by facilitating drug holidays. Its availability via the internet has increased the potential for use and abuse, although very little is known about the clinical consequences of acute intoxication. Limitations to assessing kratom toxicity include the lack of a testing methodology capable of analyzing mitragynine, the primary active alkaloid, or its metabolites in human specimens. We describe the case of a 64-year-old male with new onset seizure activity and coma following self-reported regular kratom use. Confirmation of kratom use was determined using high performance liquid chromatography coupled to an electrospray tandem mass spectrometry (HPLC-ESI/MS/MS), a novel methodology developed to quantify mitragynine in human urine. 


\section{Case Report}

A 64-year-old male was found at home by his wife unconscious and seizing. Shortly before the event, the patient had ingested a tea made with kratom and Datura stramonium. His past medical history included a colostomy repair 1 month prior as well as chronic pain and depression managed with amitriptyline, oxycodone, and kratom. His social history was significant for alcohol and tobacco use. Upon arrival by EMS, seizure activity had terminated although the patient had decorticate movement of his upper extremities. In the emergency department (ED), his Glasgow coma score was 6 with a heart rate of $110 \mathrm{bpm}$, blood pressure of $143 / 70 \mathrm{mmHg}$, temperature $98.1^{\circ} \mathrm{F}$, and respiratory rate $14 / \mathrm{min}$. His $\mathrm{SpO} 2$ was $99 \%$ on room air. Physical exam was notable for a dry oropharynx and pupils that were $4-5 \mathrm{~mm}$ and minimally reactive, with a roving conjugate gaze. He was noted to have lower extremity spasticity with manipulation. His electrocardiogram revealed a sinus tachycardia with a QRS of $100 \mathrm{~ms}$ and narrow complexes in his major limb leads. Laboratory studies were unremarkable. Acetaminophen, salicylate, and ethanol concentrations were negative. Urine drug screening was positive for cannabinoids, tricyclic antidepressants, and oxycodone.

While in the ED, approximately $1 \mathrm{~h}$ after arrival, the patient sustained a second seizure. He was intubated and given $2 \mathrm{mg}$ of lorazepam and a loading dose of $1 \mathrm{~g}$ of phenytoin. Computed tomography of the head, without contrast, was suspicious for a possible hyperdensity in the right occipital lobe and the patient was transferred to a tertiary care facility for further neurological evaluation. He arrived at the second hospital approximately $5 \mathrm{~h}$ post the inciting event. He remained unresponsive, although withdrew to noxious stimuli. Vital signs at that time included: heart rate of $96 \mathrm{bpm}$, blood pressure of 130/79 $\mathrm{mmHg}$, temperature $97.8^{\circ} \mathrm{F}$. Physical exam revealed moist oral mucosa, midsize pupils that were equal round and reactive to light, and positive bowel sounds. A serum tricyclic antidepressant level performed shortly after admission was negative (lower limit of detection: $50 \mathrm{ng} / \mathrm{mL}$ ). Magnetic resonance angiogram of the brain revealed no acute intracranial process. He remained hemodynamically stable throughout his hospital course without further seizure activity. He was extubated approximately $30 \mathrm{~h}$ post initial admission.

Upon awakening, the patient confirmed that he had consumed a kratom/Datura tea approximately $30 \mathrm{~min}$ prior to the event and that he regularly used kratom to self-medicate chronic pain. A urine specimen collected at the first hospital shortly after admission was sent to the Wadsworth Center for Laboratories and Research, NYSDOH (Albany, NY, USA) for analysis. A novel methodology was developed for mitragynine detection in human urine using HPLC-ESI/MS/ MS [1]. Good precision and accuracy were demonstrated and recoveries ranged from $80 \%$ to $115 \%$ for mitragynine extraction in urine specimens. The HPLC-ESI/MS/MS analysis data revealed excellent linear calibration for mitragynine in the range from 0.05 to $5.0 \mathrm{ng} / \mathrm{ml}$ and high precision intra-day and inter-day analysis; the method's lower limit of detection was $0.02 \mathrm{ng} / \mathrm{ml}$. The mitragynine concentration in the urine was $167 \pm 15 \mathrm{ng} / \mathrm{ml}$.

\section{Discussion}

We present a novel case of serious human toxicity following kratom use confirmed via quantitative analysis of urine by HPLC-ESI/MS/MS. Reports of toxicity secondary to kratom are rare. To date, two published cases have linked kratom use with seizures $[2,3]$, although lack of diagnostic testing in human specimens has prevented confirmatory explanation of these observed clinical effects. Boyer and colleagues reported a new onset seizure following the ingestion of kratom coadministered with modafinil [2]. This report is similar to ours in that both histories reflect accustomed kratom use that was supplemented with an additional xenobiotic, in our case Datura stramonium, resulting in the development of severe toxicity characterized by seizure activity. In the case described by Boyer et al., coma was not a concomitant finding. Roche et al. also report both seizure and coma following kratom use, although co-administered drug use/abuse was not reported.

Over 25 alkaloids have been isolated from kratom, the primary constituent of which is the indole alkaloid mitragynine [4]. Mitragynine is a known agonist of multiple receptors including the opioid receptors $\mu, \kappa, \delta$, as well as adenosine$2 \mathrm{a}$, postsynaptic alpha- 2 , dopamine- $2 \mathrm{~s}$, and various serotonin receptors [2, 5]. Additionally, 7- $\alpha$-hydroxymitragynine, a minor constituent of the plant, has been found to be a more potent opioid agonist than both mitragynine and morphine and is thought to contribute substantially to the clinical effects of kratom when ingested orally [6]. The modulation of opioid receptors and/or presynaptic adrenergic receptors may explain decreased mental status as observed in our case, although the mechanism for seizure activity remains undefined. Suggested mechanisms may involve the modification of adenosine binding or stimulation of adrenergic and/or serotonergic receptors similar to tramadol.

We are fortunate in the present case to have confirmatory testing available to definitively associate the observed symptoms with kratom use. That said, it remains a leap to infer there is causality based on an association. As this is the first biologic human specimen obtained in a clinically ill patient following kratom use, it is presently impossible to correlate a urinary concentration with clinical effects. Preliminary pharmacokinetic (PK) analysis of mitragynine in rats suggest a time to maximum concentration of 1.2-1.8 h, an elimination half-life of 3.85-9.43 h, and a volume of distribution 37.9-89.5 L/kg [7, 8]. 
Human PKs have not been studied but reported cases support relatively short-lived toxicity. It is our hope that with the advent of novel testing methodologies that this information will be forthcoming.

Limitations to our report include reliance on self-reporting for an accurate history and the inability to definitively exclude the potential for other contributory causes of toxicity. For example, it was unknown exactly how much kratom or Datura was actually ingested. Further history regarding type of kratom, how it was purchased, as well as specifics to how it was used (beyond "regular" and "excessive" daily use) were not obtained. The patient did state that he did not routinely use kratom and Datura together, although this is admittedly vague. Datura has rarely been associated with seizures [9]. Unfortunately, co-ingestion of this substance was not known until later and diagnostic testing was never pursued. The role of ethanol withdrawal should also be considered. The patient admitted to consuming two alcoholic drinks daily. Although this history is not suggestive of significant tolerance, it is possible that actual use was underreported. His ethanol level was negative and it cannot be definitively stated that the seizure activity was not a manifestation of ethanol withdrawal, albeit the profound central nervous system (CNS) depression is uncharacteristic of this syndrome without other contributory factors.

\section{Conclusion}

Presently kratom is not a controlled substance in the USA and is often promoted on the internet as a legal psychoactive product. Serious toxicity following kratom use has been associated with seizures and potentially CNS depression requiring airway support. New analytical techniques to measure mitragynine in human biologic specimens have become available recently and these methods may help aid the further study of clinical toxicity following poisoning.

\section{References}

1. Lu S, Tran BN, Nelsen JL, Aldous KM (2009) Quantitative analysis of mitragynine in human urine by high performance liquid chromatography-tandem mass spectrometry. J Chromatogr B Analyt Technol Biomed Life Sci [Internet] 24:2499-2505

2. Boyer EW, Babu KM, Adkins JE, McCurdy CR, Halpern JH (2008) Self-treatment of opioid withdrawal using kratom (Mitragynia speciosa Korth). Addiction 103(6):1048-1050

3. Roche KM, Hart K, Sangalli B, Lefberg J, Bayer M (2008) Kratom: a case of a legal high. Clin Tox 46(7):598

4. Takayama H (2004) Chemistry and pharmacology of analgesic indole alkaloids from the rubiaceous plant, mitragyna speciosa. Chem Pharm Bull (Tokyo) 52(8):916-928

5. Matsumoto K, Mizowaki M, Suchitra T, Murakami Y, Takayama H, Sakai S, Aimi N, Watanabe H (1996) Central antinociceptive effects of mitragynine in mice: contribution of descending noradrenergic and serotonergic systems. Eur J Pharmacol 317(1):75-81

6. Matsumoto K, Horie S, Ishikawa H, Takayama H, Aimi N, Ponglux D, Watanabe K (2004) Antinociceptive effect of 7 hydroxymitragynine in mice: discovery of an orally active opioid analgesic from the thai medicinal herb mitragyna speciosa. Life Sci 74(17):2143-2155

7. Janchawee B, Keawpradub N, Chittrakarn S, Prasettho S, Wararatananurak P, Sawangjareon K (2007) A high-performance liquid chromatographic method for determination of mitragynine in serum and its application to a pharmacokinetic study in rats. Biomed Chromatogr 21(2):176-183

8. de Moraes NV, Moretti RAC, Furr III EB, McCurdy CR, Lanchote VL (2009) Determination of mitragynine in rat plasma by LC-MS/ MS: application to pharmacokinetics. J Chromatogr B 877 (24):2593-2597

9. Mikolich JR, Paulson GW, Cross CJ (1975) Acute anticholinergic syndrome due to jimson seed ingestion. Ann Intern Med 83 (3):321-325 